\title{
Outcome of primary percutaneous stent-revascularization in patients with atherosclerotic acute mesenteric ischemia

\author{
Robert Forbrig, ${ }^{1,2}$, Philipp Renner ${ }^{3}$, Piotr Kasprzak ${ }^{4}$, \\ Marc-H Dahlke ${ }^{3}$, René Müller-Wille ${ }^{2}$, \\ Christian Stroszczynski ${ }^{2}$ and Peter Heiss ${ }^{2}$
}

\begin{abstract}
Background: Patients with acute mesenteric ischemia (AMI) often exhibit severe co-morbidities and significant surgical risks, leading to high perioperative morbidity.

Purpose: To investigate the feasibility of primary percutaneous stent-revascularization (PPSR) in atherosclerotic AMI and its impact on patients' outcome.

Material and Methods: Retrospective analysis of 19 consecutive patients (7 women, 12 men; median age, 69 years) with AMI caused by atherosclerotic, non-embolic stenoses/occlusions of the splanchnic arteries and PPSR. Alternative minimally invasive techniques were excluded. Clinical characteristics including the Charlson Comorbidity Index adjusted by age ( $\mathrm{CCla}$ ) and symptom duration, technical and clinical success of PPSR, clinical course, 30-day mortality, and followup were evaluated and compared to literature data for surgical approaches. Technical success was defined as residual stenosis of $<30 \%$ in diameter. Clinical success was defined as resolution of symptoms of AMl and/or normalization of serum lactate after sole PPSR.

Results: The majority of patients presented with severe co-morbidities (CCla $>4$ in 17 of 19 patients, $89 \%$ ). Median symptom duration was $50 \mathrm{~h}$. Technical and clinical success rates of PPSR were $95 \%$ (2I of 22 arteries) and $53 \%$ (I0 of 19 patients). Seven patients underwent subsequent laparotomy with bowel resection in four cases. Thirty-day mortality was $42 \%$ (8 of 19 patients).

Conclusion: In our study population of patients with atherosclerotic AMI, severe co-morbidities, prolonged acute symptoms, and significant perioperative risks PPSR of splanchnic stenoses were technically feasible and the clinical outcome was acceptable.
\end{abstract}

\section{Keywords}

Acute mesenteric ischemia, percutaneous endovascular intervention, stent-revascularization, bowel necrosis, mortality

Date received: 29 October 2015; accepted: 24 March 2016

\section{Introduction}

Stenosis of the splanchnic arteries (celiac trunk, superior mesenteric artery [SMA] and inferior mesenteric artery [IMA]) is commonly the consequence of atherosclerosis (1-3). Progressive disease may lead to insufficient bowel perfusion and symptoms of chronic mesenteric ischemia (CMI) can emerge $(2,4,5)$. In these patients, primary percutaneous stent-revascularization (PPSR) represents an already well established therapy option with high technical and clinical success rates $(6,7)$.
'Department of Neuroradiology, Ludwig-Maximilians-University Hospital Munich, Munich, Germany

${ }^{2}$ Department of Radiology, University Medical Center Regensburg, Regensburg, Germany

${ }^{3}$ Department of Surgery, University Medical Center Regensburg, Regensburg, Germany

${ }^{4}$ Department of Vascular Surgery, University Medical Center Regensburg, Regensburg, Germany

Corresponding author:

Robert Forbrig, Department of Neuroradiology, Ludwig-MaximiliansUniversity Hospital Munich, Marchioninistr. 15, 81377 Munich, Germany. Email: robert.forbrig@med.uni-muenchen.de 
Due to further vessel narrowing and/or periods of arterial hypotension, symptoms of acute mesenteric ischemia (AMI) may occur $(1,2,5)$. In case of persistent insufficient bowel perfusion, reversible bowel ischemia progresses to irreversible necrosis (8). The standard therapy of AMI is surgical revascularization of splanchnic arteries by means of arterial bypass grafting or thromboembolectomy and resection of necrotic bowel segments $(2,8,9)$. This approach requires laparotomy and may be challenging in patients with severe atherosclerosis, prior abdominal surgery, peritonitis, and/or significant co-morbidities $(1,2,9-12)$. To overcome the surgical risks and morbidity, minimally invasive, endovascular approaches including intraoperative retrograde stenting of the SMA as well as percutaneous revascularization are increasingly performed $(1,2,5,13-21)$.

The purpose of this study was to investigate the feasibility of PPSR in patients with AMI, caused by atherosclerotic stenoses/occlusions (but not embolic occlusions) of the splanchnic arteries, and its impact on the clinical outcome.

\section{Material and Methods}

\section{Data collection}

At the University Medical Center Regensburg, a total of 19 consecutive patients ( 7 women, 12 men; median age, 69 years; age range, 50-88 years) with AMI underwent PPSR of atherosclerotic stenoses of the splanchnic arteries between January 1999 and February 2011. Embolic occlusions - representing another common cause of AMI where the embolus is usually located at least $3 \mathrm{~cm}$ distal from the ostium - were not included in this survey. Alternative minimally invasive approaches (e.g. transluminal aspiration (13)) and primary laparotomy were excluded as well.

We retrospectively analyzed the patients' medical records and imaging data focusing on clinical characteristics including the extent of co-morbidities and time interval between onset of acute symptoms and PPSR, the technical and clinical success of PPSR, the clinical course including need of subsequent laparotomy and bowel resection, and 30-day mortality as well as follow-up.

An institutional review board approval was not required for publishing a retrospective study. The principles of the Declaration of Helsinki were followed. All patients and/or their relatives gave informed consent prior to inclusion in this study.

\section{Definition of AMI}

AMI was present if: (i) the patients showed typical signs and symptoms of AMI (persistent abdominal pain, gastrointestinal bleeding, clinical peritonitis, pneumatosis intestinalis, portal venous gas, shock, and/or elevated serum lactate); (ii) at least one splanchnic artery showed a stenosis of $>50 \%$ in diameter; and (iii) there was no other disease that accounted for the symptoms (6).

\section{Co-morbidities}

The Charlson Comorbidity Index adjusted by age (CCIa) (22) was used in order to compare the survival with current literature data of primary open surgical approach considering the extent of co-morbidities and the patients' age.

\section{Laboratory, imaging, and interventional data}

The serum values of C-reactive protein (CRP), white blood cell (WBC) count, and lactate temporally nearest to PPSR were documented.

Contrast-enhanced arterial and portal venous phase computed tomography (CT) scans of the abdomen and pelvis were acquired. CT images were reconstructed in the axial and coronal planes (3- $\mathrm{mm}$ slice width/3-mm intervals). Diagnostic digital subtraction angiography (DSA) started with an anterior-posterior abdominal aortography in order to visualize the flow dynamics and potential collateral perfusion of the splanchnic arteries. Subsequently, selective lateral images of the stenotic splanchnic arteries were carried out.

CT and DSA data were read in consensus by two radiologists with 5 (FR) and 12 (HP) years of experience in body imaging. Based on visual grading of the splanchnic arteries, a stenosis of $51-70 \%$ and $71-99 \%$ in diameter was defined as moderate and high-grade stenosis, respectively. Thickness of the bowel wall of $>3 \mathrm{~mm}$ was considered pathologic (23) and signs of AMI (non-enhancing bowel wall, pneumatosis intestinalis, portal venous gas, free intraperitoneal air) were documented. Furthermore, findings of endoscopy were supplemented. Concerning interventional data analysis, the duration of PPSR, the kind and size of implanted stents, technical and clinical success as well as peri-interventional complications were assessed. Technical success (based on intention-to-treat) was defined as successful completion of the intervention and a residual stenosis of $<30 \%$ in diameter $(5,17)$. Clinical success was defined as resolution of symptoms of AMI and/or normalization of serum lactate after sole PPSR.

\section{Decision-making process}

Each case was discussed interdisciplinary by a general surgeon, a vascular surgeon, and an interventional radiologist. In detail, the pros and cons of primary surgical revascularization and PPSR were evaluated, taking into account the patients' general condition, co-morbidities, 
and other surgical risk factors such as severe splanchnic calcifications and prior laparotomies with potential abdominal adhesions. In each case, the physicians and the patient and/or their relatives decided in consensus to attempt PPSR. Subsequent laparotomy was indicated if signs or symptoms of AMI persisted, recurred, or got worse.

\section{Endovascular technique}

In four $(21 \%)$ and 15 of 19 patients $(79 \%)$ a brachial and femoral access, respectively, was chosen. If the SMA was stenotic or occluded, the primary aim was to revascularize this artery. If an occluded SMA could not be revascularized and a stenosis of the celiac trunk and/or the IMA was identified, it was attempted to revascularize primarily the celiac trunk and alternatively the IMA in order to improve perfusion of the small bowel via arterial collaterals (e.g. via pancreatic arcades). Prior to selective catheterization of the splanchnic arteries, 2500 or 5000 units of heparin were administered, dependent on the interventionist's preference and history of recent gastrointestinal bleeding. Then, the tip of a 5-7 French sheath was carefully advanced through the stenosis. In case of severe or circular calcification, the stenosis was dilated with, for example, a $3 \mathrm{~mm}$ balloon prior to sheath advancement. The interventionist inserted a stent of his preference considering the diameter of the treated artery. If completion angiography depicted a residual stenosis of $>30 \%$, balloon angioplasty was performed or a second stent was implanted. To prevent acute in-stent thrombosis prior to stent release, $100 \mathrm{mg}$ or $250 \mathrm{mg}$ acetylsalicylic acid were administered according to the interventionists' preference considering recent gastrointestinal bleeding. To prevent in-stent neointimal hyperplasia platelet aggregation inhibition was continued with $100 \mathrm{mg}$ acetylsalicylic acid per day lifelong. As soon as the patient was in a stable condition without need of additional operative treatment, $75 \mathrm{mg}$ clopidogrel per day for at least 6 weeks were administered.

\section{Results}

Details of the 19 patients with PPSR are summarized in Supplementary Table 1 (available online).

\section{Co-morbidities}

Each of the 19 patients exhibited at least one co-morbidity (median, 3; range, 1-7). The median CCIa score was 6 (range, 3-17). Moderate to severe renal disease (12 of 19 patients, $63 \%$ ) and peripheral vascular disease (10 of 19 patients, $53 \%$ ) were the most prevalent comorbidities.

\section{Abdominal symptoms, laboratory parameters, and imaging findings}

Three patients were not responsive and/or anesthetized (patients 5, 6, and 18). Twelve of the remaining 16 patients $(75 \%)$ reported persistent abdominal pain. Two of 19 patients $(11 \%)$ exhibited clinical peritonitis (patients 6 and 7) and seven of 19 patients (37\%) suffered from gastrointestinal bleeding. Median time interval between onset of symptoms of AMI and/or elevation of lactate and PPSR was $50 \mathrm{~h}$ (range, $8 \mathrm{~h}-12$ days). Five patients reported history of CMI (patients $2,8,9,12$, and 16).

Median levels of CRP, WBC count, and lactate were $124 \mathrm{mg} / \mathrm{L}$ (range, 9-301 mg/L), 13/nL (range, 6-32/nL), and $20 \mathrm{mg} / \mathrm{dL}$ (range, $10-68 \mathrm{mg} / \mathrm{dL}$ ), respectively. The levels of CRP, WBC count, and lactate were increased above normal in 14 of $14(100 \%), 11$ of $17(65 \%)$ and six of 12 patients $(50 \%)$ with available laboratory data, respectively.

CT showed pneumatosis intestinalis in three cases (patients 6, 7, and 14) including gas within the portal veins in one participant (patient 14). The bowel wall was pathological thickened in nine patients. Endoscopy depicted gastric ischemia and ischemic colitis in one (patient 6) and five patients (patients 2, 8, 13, 15 , and 17), respectively.

\section{Angiographic findings and PPSR}

Based upon DSA and CT findings, stenosis or occlusion was situated within the proximal $3 \mathrm{~cm}$ of the splanchnic artery. The etiology of the splanchnic stenosis/occlusion was most likely an atherosclerotic plaque with or without thrombosis in each case.

In two (patients 9 and 17) and one (patient 15) cases simultaneous stenting of the celiac trunk and SMA as well as simultaneous stenting of the SMA and IMA were carried out. In four of 22 arteries $(18 \%)$, two stents were inserted into one artery. Median duration of PPSR was $80 \mathrm{~min}$ (range, 20$200 \mathrm{~min}$ ). In total, 21 of 22 arteries were successfully revascularized resulting in a technical success rate of $95 \%$.

One peri-interventional complication occurred: in patient 12 it was attempted to revascularize an occlusion of the celiac trunk via the left brachial access. The patient was limited cooperative and angiographic image quality was significantly reduced due to motion artifacts and obesity. After several attempts it was considered that the celiac trunk was successfully catheterized and two stents were placed at the site of the presumed stenosis. Control angiography showed that the stents were erroneously implanted into the SMA. 


\section{Clinical course and follow-up}

Post-interventionally, in 10 of 19 patients the symptoms of AMI resolved and/or serum lactate normalized without need of further specific therapy, leading to a clinical success rate of $53 \%$.

Seven of the nine patients without clinical success $(78 \%)$ underwent subsequent laparotomy with bowel resection in four patients after a median time interval of $3.5 \mathrm{~h}$ (range, $1 \mathrm{~h}-18$ days). One of these patients survived (patient 9).

The overall 30-day mortality was $42 \%$ (8 of 19 patients). The three patients with pneumatosis intestinalis died. The 30-day mortality of patients with at least one post-interventionally occluded splanchnic artery was $75 \%$ (6 of 8 patients).

Eleven of 19 patients $(58 \%)$ survived the 30-day post-interventional period, including four of five patients $(80 \%)$ with a history of CMI, and were discharged from hospital after a median stay of 4 days. None of the surviving patients exhibited short-bowel syndrome during a median follow-up of 43 months (range, 1-81 months).

\section{Discussion}

Unfortunately, mortality of patients with AMI remains high $(8,20,21,24)$ as diagnosis and therapy are often delayed $(8,25)$, the majority of patients presents with several co-morbidities $(1,5,21,26)$, and open revascularization is still a major trauma - particularly in patients with increased surgical risks $(5,9-12)$.

Recent data show overall mortality rates of about 30 $50 \%$ for patients with AMI undergoing traditional open surgery $(20,21,27-29)$. Here, duration of acute symptoms of more than $24 \mathrm{~h}$ was associated with a further increase of mortality to $70-80 \%(21,27,28)$. In comparison, in the present study the overall 30-day mortality was lower $(42 \%)$ with a median time from onset of symptoms of $50 \mathrm{~h}$. Also, Arthurs et al. reported prolonged symptom duration for patients treated by an endovascular approach $(62 \mathrm{~h})$ with significantly lower mortality when compared to open surgery (20).

We believe that not only duration of symptoms and the choice of treatment, but also other factors such as the rate of residual bowel perfusion, due to incomplete vessel occlusion or capable collateral circulation, have additional influence on overall outcome $(13,14)$. This is supported by the observation that most of our patients with a history of CMI survived the post-interventional period ( 4 out of 5 patients).

The impact of co-morbidities was further documented by Marchena-Gomez et al. (26) who analyzed patients with AMI undergoing open surgery. In their study, the perioperative mortality of patients with a CCIa score of greater than 4 was $72 \%$ (26). In the present study, the majority of patients $(89 \%)$ exhibited a CCIa score of greater than 4 and the 30-day mortality was as low as $42 \%$. Hence, patients with severe comorbidities might particularly benefit from PPSR. Other endovascular studies reported mortality rates below 30\% $(1,14,21)$. However, only few data exist on PPSR only in atherosclerotic AMI, and several of our patients were considered inoperable. Thus, the clinical outcome of our study population is clearly in keeping with what has been observed by other authors.

Although we favor the endovascular approach as described in this report, the following drawbacks of PPSR have to be taken into account carefully: (i) PPSR might delay resection of necrotic bowel. A hybrid surgical room with facilities for both percutaneous and open surgical intervention can save valuable time (13). This technique was not used in our center for the here reported group. (ii) Percutaneous revascularization of (chronically) occluded arteries can be technically challenging. In our study population eight patients had at least one chronic splanchnic occlusion, none of these vessels were revascularized percutaneously and 30-day mortality was high for this subgroup of patients $(75 \%)$. Surgical revascularization of occluded arteries might have improved outcome $(1,4)$. (iii) Pneumatosis intestinalis indicates advanced AMI and is usually associated with transmural bowel necrosis (30). In the present study three patients with pneumatosis intestinalis underwent PPSR. All three patients died. In another study two patients with pneumatosis intestinalis also received primary percutaneous treatment and died (17). Therefore, we currently believe that immediate laparotomy is indicated for patients with pneumatosis intestinalis. (iv) Three of eight patients dying within 30 days $(38 \%)$ suffered from cardiac complications. In these three patients, inflammatory molecules of the bowel possibly entered circulation and induced ischemia-reperfusion injury (13). Hence, cardiac complications must be carefully avoided when possible.

We believe that our current retrospective study can contribute to hypothesis generation in the field of intestinal reperfusion by the endovascular route. However, the following limitations must be taken into account: (i) there is a potential selection bias towards patients with a higher rate of co-morbidities, increased surgical risks, and in whom PPSR of at least one splanchnic artery was considered possible; (ii) data of time to intervention, balloon dilatation prior to stent placement, administration of acetylsalicylic acid during intervention as well as duration of clopidogrel administration post-intervention could not be fully obtained due to the retrospective nature of the study; and (iii) the study population is clearly limited in number due to the novelty of the approach. Further prospective trials with larger sample sizes are needed.

In conclusion, in our study population of 19 selected patients with atherosclerotic AMI presenting with severe 
co-morbidities (CCIa $>4$ in $89 \%$ ), prolonged acute symptoms (median, $50 \mathrm{~h}$ ) as well as significant surgical risks, PPSR of splanchnic stenoses was technically feasible. Clinical outcome was acceptable overall (30-day survival $58 \%$ ), although splanchnic occlusions and signs of bowel necrosis were associated with worse outcome.

\section{Supplementary material}

Supplementary material is available online at acr.sagepub. com/supplemental.

\section{Declaration of conflicting interests}

The author(s) declared no potential conflicts of interest with respect to the research, authorship, and/or publication of this article.

\section{Funding}

The author(s) received no financial support for the research, authorship, and/or publication of this article.

\section{References}

1. Acosta S, Björck M. Modern treatment of acute mesenteric ischemia. Br J Surg 2014;101:e100-108.

2. Renner P, Kienle K, Dahlke MH, et al. Intestinal ischemia: current treatment concepts. Langenbecks Arch Surg 2011; 396:3-11.

3. Park WM, Gloviczki P, Cherry KJ Jr, et al. Contemporary management of acute mesenteric ischemia: Factors associated with survival. J Vasc Surg 2002;35:445-452.

4. Sharafuddin MJ, Nicholson RM, Kresowik TF, et al. Endovascular recanalization of total occlusions of the mesenteric and celiac arteries. J Vasc Surg 2012;55:1674-1681.

5. Blauw JT, Meerwaldt R, Brusse-Keizer M, et al. Retrograde open mesenteric stenting for acute mesenteric ischemia. J Vasc Surg 2014;60:726-734.

6. Heiss P, Zorger N, Kaempfe I, et al. Stenting in the treatment of chronic mesenteric ischemia: technical and clinical success rates. Rofo 2008;180:906-914.

7. Aksu C, Demirpolat G, Oran I, et al. Stent implantation in chronic mesenteric ischemia. Acta Radiol 2009;50:610-616.

8. Oldenburg WA, Lau LL, Rodenberg TJ, et al. Acute mesenteric ischemia: a clinical review. Arch Intern Med 2004;164:1054-1062.

9. Eckstein HH. Die akute mesenteriale Ischämie. Resektion oder Rekonstruktion. [Acute mesenteric ischemia. Resection or reconstruction?]. Chirurg 2003;74:419-431.

10. Gore RM, Yaghmai V, Thakrar KH, et al. Imaging in intestinal ischemic disorders. Radiol Clin North Am 2008;46:845-875.

11. Debus ES, Diener H, Larena-Avellaneda A. Akute intestinale Ischämie. [Acute intestinal ischemia]. Chirurg 2009; 80:375-385.

12. Luther B. Akute viszerale Ischämie. [Acute visceral ischemia]. Gefässchirurgie 2006;11:167-172.

13. Heiss P, Loewenhardt B, Manke C, et al. Primary percutaneous aspiration and thrombolysis for the treatment of acute embolic superior mesenteric artery occlusion. Eur Radiol 2010;20:2948-2958.
14. Acosta S, Sonesson B, Resch T. Endovascular therapeutic approaches for acute superior mesenteric artery occlusion. Cardiovasc Intervent Radiol 2009;32:896-905.

15. Hawkins BM, Khan Z, Abu-Fadel MS, et al. Endovascular treatment of mesenteric ischemia. Catheter Cardiovasc Interv 2011;78:948-952.

16. Schoots IG, Levi MM, Reekers JA, et al. Thrombolytic therapy for acute superior mesenteric artery occlusion. J Vasc Interv Radiol 2005;16:317-329.

17. Gagnière $\mathbf{J}$, Favrolt $\mathrm{G}$, Alfidja A, et al. Acute thrombotic mesenteric ischemia: primary endovascular treatment in eight patients. Cardiovasc Intervent Radiol 2011;34: 942-948.

18. Ryer EJ, Kalra M, Oderich GS, et al. Revascularization for acute mesenteric ischemia. J Vasc Surg 2012;55:1682-1689.

19. Block TA, Acosta S, Björck M. Endovascular and open surgery for acute occlusion of the superior mesenteric artery. J Vasc Surg 2010;52:959-966.

20. Arthurs ZM, Titus J, Bannazadeh M, et al. A comparison of endovascular revascularization with traditional therapy for the treatment of acute mesenteric ischemia. J Vasc Surg 2011;53:698-705.

21. Beaulieu RJ, Arnaoutakis KD, Abularrage CJ, et al. Comparison of open and endovascular treatment of acute mesenteric ischemia. J Vasc Surg 2014;59:159-164.

22. Charlson ME, Szatrowski TP, Peterson J. Validation of a combined comorbidity index. J Clin Epidemiol 1994;47: 1245-1251.

23. Schaefer-Prokop C, Jörgensen M, Galanski M. Gastrointestinaltrakt. In: Prokop M, Galanski M, Schaefer-Prokop C, et al. (eds) GanzkörperComputertomographie. Stuttgart, New York: Thieme, 2007, pp.573-577.

24. Schoots IG, Koffeman GI, Legemate DA, et al. Systematic review of survival after acute mesenteric ischemia according to disease aetiology. Br J Surg 2004;91:17-27.

25. Acosta-Merida MA, Marchena-Gomez J, HemmersbachMiller M, et al. Identification of risk factors for perioperative mortality in acute mesenteric ischemia. World J Surg 2006;30:1579-1585.

26. Marchena-Gomez J, Acosta-Merida MA, HemmersbachMiller M, et al. The age-adjusted Charlson Comorbidity Index as an outcome predictor of patients with acute mesenteric ischemia. Ann Vasc Surg 2009;23:458-464.

27. Kougias P, Lau D, El Sayed HF, et al. Determinants of mortality and treatment outcome following surgical interventions for acute mesenteric ischemia. J Vasc Surg 2007; 46:467-474.

28. Aliosmanoglu I, Gul M, Kapan M, et al. Risk factors effecting mortality in acute mesenteric ischemia and mortality rates: a single center experience. Int Surg 2013;98:76-81.

29. Duran M, Pohl E, Grabitz K, et al. The importance of open emergency surgery in the treatment of acute mesenteric ischemia. World J Emerg Surg 2015;10:45.

30. Wiesner W, Mortele KJ, Glickman JN, et al. Pneumatosis intestinalis and portomesenteric venous gas in intestinal ischemia: correlation of CT findings with severity of ischemia and clinical outcomes. Am J Roentgenol 2001;177:1319-1323. 